\title{
OBSTACLES, SOLUTIONS AND APPLICATIONS FOR THE DEVELOPMENT OF MODERN WIRELESS NETWORKS
}

\author{
Márk KOVÁCS, ${ }^{1}$ Zsolt Csaba JOHANYÁK² \\ John von Neumann University, GAMF Faculty of Engineering and Computer Science, Kecskemét, Hungary \\ ${ }^{1}$ kovacs.mark@gamf.uni-neumann.hu \\ ${ }^{2}$ johanyak.csaba@gamf.uni-neumann.hu
}

\begin{abstract}
This study describes 5G, the latest wireless technology that is currently under development. It will ensure increased bandwidth as well as newer and higher quality antennas. 5G is actually about further developing 4G/LTE. Due to the rapidly growing number of network devices, the current LTE technology will soon be unsatisfactory in terms of quality of service (QoS), therefore a new concept is needed.

The solution to this problem depends on the quality and complexity of the antennas, as well as traffic management. The planned Fifth Generation Network focuses on these issues to provide more accessible, faster, and more reliable services. The new technology will offer a lot of opportunities for IoT compatible devices such as self-driving vehicles or those used in healthcare. In our opinion we will soon achieve a world of unlimited Internet access.
\end{abstract}

Keywords: 5G, latency, small cell, MIMO, Full Duplex.

\section{Introduction}

The first generation of wireless networks appeared in the early 1970s with only 2.4 Kbps data transfer rate, and was used only for making calls. $2 \mathrm{G}$ was reliable and uses digital signals, which allowed the use of well-known SMS and e-mail services. With the third generation, data transfer speed reached up to $21.6 \mathrm{Mbps}$, thereby improving the sound quality, as well as allowing the first video-conferences. However, this network was still too slow and too expensive.

The current 4G networks were introduced in 2010, with major advances in 3rd generation networks. Bandwidth has reached a level where media content can be transferred in enjoyable quality. However, as the number of users increases, the technology needs to be further developed. However, a further problem arose when using 4G: the battery life of the device was significantly reduced. [1][2]

\section{4G/LTE networks}

\subsection{Formation}

The construction and use of fourth generation networks began in 2010. 4G is much more cost-effective than its predecessors. The data transfer rate has increased, averaging around $100 \mathrm{Mbps}$, but it can reach up to $1 \mathrm{Gbps}$ as well. This bandwidth already enables the transmission of high quality content.

Here the main novelty is the LTE-A (Long Term Evolution Advanced) technology, which is a further developed version of LTE, i.e. a step towards $5 \mathrm{G}$.

Mobile networks are often fragmented, not using a certain frequency band, which slows down data transmission. This issue is solved by the socalled carrier aggregation that merges up to 20 channels from one spectrum into one stream.

The further developed version of LTE-A is the so called MIMO (Multiple Input Multiple Output) technology, which allows devices to use twice as many antennas to achieve higher data rates. [3] 


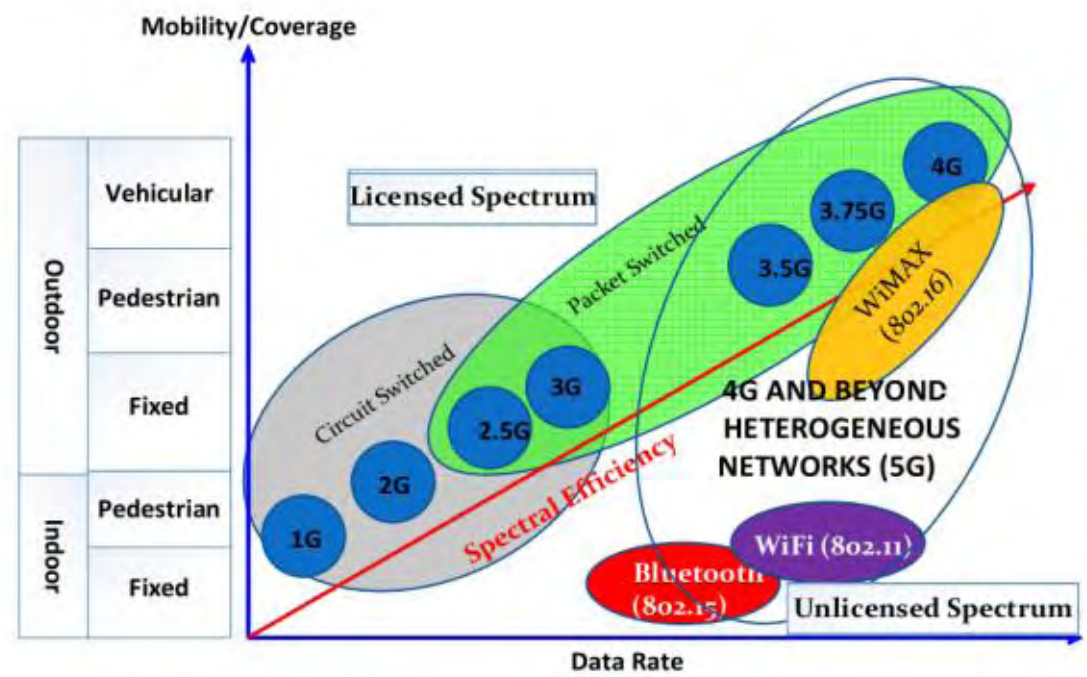

Figure 1. Process of wireless technology development [4]

\subsection{Market share}

By now, $4 \mathrm{G}$ networks have a significant market share (39\%) worldwide [5]. Even the simplest smart phones already support at least the standard LTE technology, but most smart devices support the two times faster LTE-A technology as well. All Hungarian mobile operators ensure a nationwide full coverage of the fourth-generation network.

\subsection{Shortcomings}

Due to the rapidly growing number of smartphones and other network devices, the current network will not be able to serve clients fast enough. In addition, it will be increasingly important to have less delay time and greater coverage.

\section{5G wireless networks}

\subsection{Millimeter waves}

Currently, network devices operate in a frequency spectrum that is already beginning to be too noisy and crowded due to the increasing number of devices and this can lead to poor quality of service. $5 \mathrm{G}$, the so-called millimeter wave communication, introduces new frequency ranges into telecommunications. The range of millimeter waves can be up to $300 \mathrm{GHz}$. These sections have not been used before, so they are not noisy.
However, their disadvantage is that these waves cannot pass through the wall or other obstacles.

\subsection{Small cells and massive MIMO}

In order to prevent obstacles from affecting the propagation of waves, many small towers must be built close together to avoid obstacles. When moving, the device must be able to switch automatically from one cell to the next.

MIMO has multiple inputs and outputs. Several antennas can be found at a base station to handle cellular data traffic. While standard MIMO networks use two or four antennas, today, thanks to newer developments, there are already many more antennas for a base station, increasing coverage and response time.

By using an increasing number of antennas, the device can receive and send information from all directions. However, this requires the so-called beamforming, which is a kind of signalling lamp that ensures that a particular user is sent focused data. It is a much more effective method because it can send and receive more data at once. [6]

\subsection{BDMA (Beam Division Multiple Access)}

The currently used communication systems apply two types of technology: Time Division Multiple Access (TDMA), and Frequency Division Multiplex Access (FDMA) technology. Unfortunately, none of them is perfect because of timesharing

TDMA shares a certain frequency band, which means that users receive the signal only intermittently, and this can result in a slowdown in traffic. 
In a few years the number of mobile users may be multiplied, making it difficult to build a well-functioning technology.

In the FDMA solution, the channel bandwidth is divided into independent subchannels and each user receives one of them. However, this solution is not very efficient because of the traffic fluctuation, i.e. it can have unused and overloaded channels at the same time.

Thus the two currently used technologies need to be replaced with a better method. The Beam Division Multiple Access (BDMA) [7] technology is an attempt to solve this problem. The proposal, developed by Korean engineers, is independent of frequency and time. In this technology, the base station assigns a separate shield to each mobile station, which only occurs after evaluating the current position of the device and the speed of movement. If there are more mobile devices at a similar angle, they can share one broadcast with TDMA. This solution is more effective because it allocates resources more efficiently among users [8].

\section{Comparing local wired and wireless networks}

The Fifth Generation can provide such a breakthrough in wireless communication that it may later lead to the obsolescence of local wired networks.

In current widespread local area networks the $2.4 \mathrm{GHz}$ frequency band Wi-Fi is becoming overloaded, and becoming busier if more people want to use it at the same time. The newly introduced $5 \mathrm{GHz}$ high frequency band wireless standard provides more reliable speed and availability for multiple users. The only drawback of the $5 \mathrm{GHz}$ network is that it has a smaller range, but it is not

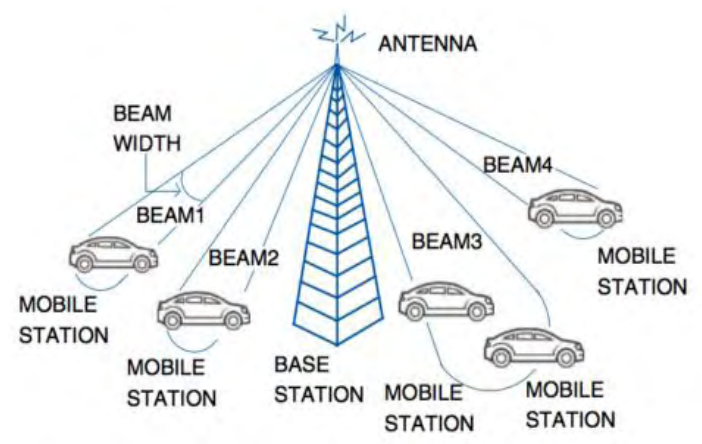

Figure 2. Beam Division Multiple Access [8] a significant factor because it is usually used in small places, at home or in the office. [9]

With the introduction of $5 \mathrm{G}$, it is expected that we will be able to connect to the $5 \mathrm{G}$ network wirelessly with the $5 \mathrm{GHz}$ standard, thus almost eliminating wired networks. There is already a similar solution for some service providers, using a socalled MIFI portable Wi-Fi hotspot device which according to our current LTE-A networks, works well.

In our view, this technology will spread to wired networks in the future, not only in local networks but also worldwide which is helping to develop WWWW (World Wide Wireless Web). Although fiber technology would be a good solution and $t$ would be a big step forward in terms of speed, its full cost is huge, while $5 \mathrm{G}$ promises better conditions.

\section{5G and the Internet of Things}

At the age of Industry 4.0, new developments are expected in many areas of IoT. 5G uses Direct Device-to-Device communication to help develop IoT. In addition, direct connection between devices can greatly reduce the delay time.

Self-driving car sensors generate a huge amount of data, such as temperature, weather, current GPS position, or where an accident occurred, and where there may be obstacles in traffic. Processing this data takes a lot of energy. The higher speed and the low delay time make it possible for these data to be continuous at all times, and to make good decisions about the vehicle on a basic basis, and to be able to inform the similar smart vehicle in circulation in time. This quick decision-making capability is a great necessity because a fast response is required in a given situation in order to make the right decision.

$5 \mathrm{G}$ can also offer great opportunities for healthcare. The so-called "Internet of Medical Things" means new technologies such as clinical portable devices, remote sensors and other medical devices that allow physicians to remotely monitor a patient's condition. By analysing the data, it may be easier to track the diagnosis and the effect of the selected treatment. In addition, the spread of telemedicine can be achieved by providing high quality video conferencing. [10]

\section{Conclusions}

Once the plans are implemented, the deployment of fifth generation networks can begin after 
2020. The data transfer rate will increase enormously, which can range from a minimum of 1 Gbps to tens of Gbps, which means that for example an HD movie can be downloaded in a few seconds. It was only in laboratory conditions but in autumn 2017 Magyar Telekom and Ericsson reached 22 gigabytes of download speeds in $\mathrm{Bu}$ dapest. With the promised delay of around $1 \mathrm{~ms}$, there is a lot of progress in many areas.

In this article, we did not discuss a factor that can be of great importance, i.e. how much this new technology would affects our health. Antennas that are closer to each other mean greater radiation than before, and so there could arise unknown health risks as well.

\section{Acknowledgement}

This research is supported by EFOP-3.6.1-16-201600006 "The development and enhancement of the research potential at John von Neumann University" project. The Project is supported by the Hungarian Government and cofinanced by the European Social Fund.

\section{References}

[1] Majid I. B., Naira N., Insha M., Kamran A. N., Suhaib A.: Evolution of Mobile Wireless Communication Systems from $1 G$ to $5 G$. A Comparative Analysis. International Journal of Scientific Research in Computer Science, Engineering and Information Technology, 4/1. (2018) 1-8.
[2] Abhishek Gupta, Bhavesh Mishra: A Survey on Wireless Technology 5G. International Journal of Innovative Research in Computer and Communication Engineering, 2016/9. 16330-16337.

[3] Gopal B. G., Kuppusamy P. G.: A Comparative Study on $4 G$ and $5 G$ Technology for Wireless Applications. IOSR Journal of Electronics and Communication Engineering, 10/6, (2015), 67-72.

[4] Akhil G., Rakesh K. J.: A Survey of $5 G$ Network: Architecture and Emerging Technologies, IEEE Access, 3. (2015) 1206-1231.

[5] LTE Achieves: 39\% Market Share Worldwide. (accessed: 18 February 2019)

http://www.5gamericas.org/en/newsroom/ press-releases/lte-achieves-39-market-shareworldwide/

[6] Sagarkumar P., Harshad P., Shivam Shah: Review On $5 G$ Wireless Technology. IJSRCSEIT, 3/3. (2018) 1618-1621.

[7] Beam division multiple access system and method for mobile communication system: Patent US20100165914A1 (accessed: 18 February 2019) h t tps://patents.google.com/patent/ US20100165914A1/en

[8] Electronicsforu: How Does BDMA Technology Work in 5G Network?, 2018. (letöltve: 2019.02.3.). https://electronicsforu.com/resources/lern-electronics/bdma-technology-5g-network

[9] Verma L., Fakharzadeh M., Choi S.: Wifi on steroids: 802.11AC and 802.11AD. IEEE Wireless Communications, 20/6. (2013) 30-35. https://doi.org/10.1109/mwc.2013.6704471

[10] Darrell M. West: How $5 G$ technology enables the health internet of things. B |Center for Technology Innovation at BROOKINGS, July 2016. 